\title{
Visual form perception: Congruence among spatial configurations'
}

\author{
LEONA S. AIKEN AND D. R. BROWN ${ }^{2}$ \\ PURDUE UNIVERSITY
}

\begin{abstract}
Multidimensional scaling (MDS) techniques were used to obtain spatial solutions. for judgments of similarity and discrimination difficulty between pairs of random polygons. For both tasks, it was possible to psychophysically predict the three dimensions of the final solutions using physical measures of the forms. These solutions were compared to each other and to a spatial analysis of discrimination difficulty measured in visual noise (Aiken \& Brown, 1968) using canonical correlation techniques. Although the two judgment tasks were highly similar, it was concluded that neither was similar to the discrimination-in-noise solution.
\end{abstract}

Shepard (1960) discussed specific methods of similarity measurement which may provide experimental data from which a spatial representation of perceived relations among stimuli may be generated. In general, procedures yielding estimates of interpoint distances among stimuli may be divided into two classes. First are the judgmental tasks of which similarity estimates are the most common, and second are experimental tasks from which distance estimates are derived. Shepard (1960) suggested, for instance, that amplitude of a generalized conditioned response, percentage of errors in a paired-associates task, discrimination latency, and sorting time could provide the basis for generating interpoint distances in a spatial configuration. While perceived similarity based upon judgments has been the focus of most multidimensional scaling (MDS) research and is of interest in its own right, the implication has been that perceived similarity is a general construct which underlies performance in a variety of situations, and that data based upon a variety of operations would converge upon the common construct of psychological distance among stimuli. The importance of this notion can hardly be overemphasized in view of the fact that most stimulation of interest in studies of perception, learning, and motivation is physically multidimensional. It seems naive at this point in time to assume that performance is unidimensionally related to "stimuli" without an attempt to study subjective evaluation of stimulation represented spatially.

In a series of studies, we have been attempting to carry out the suggestion (Brown \& Owen, 1967) of using MDS procedures to develop the psychophysical study of visual form perception and, at the same time, to examine the question of a common perceptual space for a variety of experimental operations. In earlier studies highly congruent spaces were obtained when MDS results of judged similarity (Behrman \& Brown, 1968) and discrimination latency (Brown \& Andrews, 1968) were compared for a common set of forms, and it was interesting to note that these dimensions were similar to those found to be useful for discrimination in other studies (Forsyth \& Brown, 1967, 1968) and similar to MDS dimensions of similarity obtained elsewhere (Stenson, 1968). Recently (Aiken \& Brown, 1968) interpretable MDS results were obtained when the dependent variable in a discrimination task was the amount of noise present when a discrimination between forms was accurately accomplished. This study was designed to examine the congruence of the latter data with spatial analyses based upon two judgment tasks, judged similarity and judged discrimination difficulty.

\section{Method}

\section{EXPERIMENT 1}

Subjects. Twelve college students, six male and six female, were paid for their participation. ${ }^{3}$

Stimuli. Twenty, eight-sided random polygons were selected from a sample of 200 such forms which were generated by a modified Method 1 of Attneave and Arnoult (1956). These forms were selected to represent the range of five physical measures which were highly correlated with five factors obtained when 80 physical measures were factored for the sample of 200 forms (Brown \& Owen, 1967). Forms were also selected so that the linear correlation among the five measures was minimized $(-.18$ to +.34$)$ for this sample. These forms were used in an earlier study (Aiken \& Brown, in press) and are shown in Fig. 1 with standardized measures.

Procedure. The ( $\left.\begin{array}{l}n \\ 2\end{array}\right)$ pairs (190) of forms were back-projected on a screen and $S$ spoke a seven-point similarity rating into a microphone which extinguished the projector. For each S, pairs were presented in blocks of 50 trials, in the order specified by Ross (1934), over a five-day period. The 50 trials on the first day were practice trials. Order of presentation of the four blocks of 50 trials was controlled by assigning four groups of three Ss each to the rows, days to the columns, and blocks to the entries of a 4 by 4 Latin square. Ten pairs, selected at random across the four days for each $\mathrm{S}$, were repeated to evaluate reliability.

$$
\begin{aligned}
& \text { MEASURE }
\end{aligned}
$$

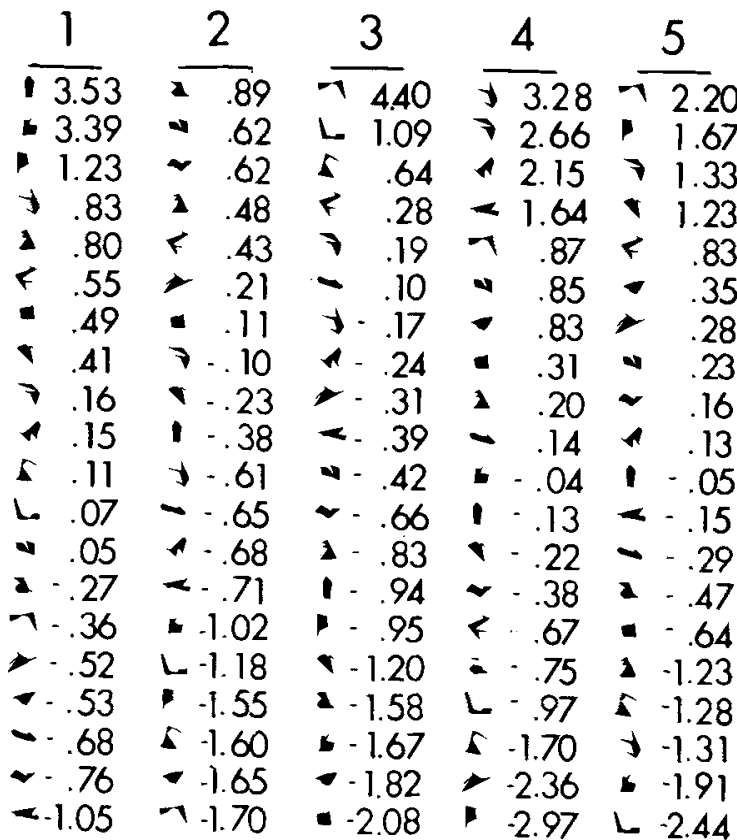

Fig. 1. Ordering of shapes on five selection measures: (1) second moment of combined radials; (2) third moment of interior angles; (3) ratio of vertical to horizontal extent; (4) $\mathrm{Y}$ coordinate of areal center of gravity; (5) X coordinate of areal center of gravity. See Brown and Owen (1967) for a more complete description of these measures. 


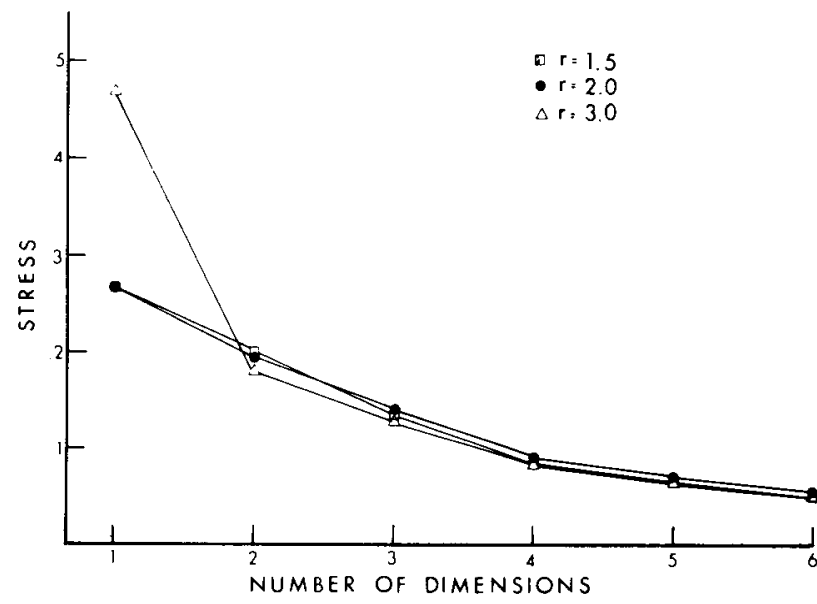

Fig. 2. Values of stress for the three Minkowski solutions $(r=1.5,2.0$, and 3.0 ) to the similarity judgments.

\section{Results}

The mean reliability coefficient (Pearson, product moment) for the 10 repeated trials was $.86(p \leqslant .01)$ supporting the conclusion that the basic judgments were highly reliable.

Judgments averaged over Ss served as input for the Kruskal nonmetric MDS analysis (Kruskal, 1964a, 1964b). Analyses were completed in one through six dimensions using Minkowski $\mathrm{r}$ metrics of 1.5, 2.0, and 3.0. Goodness of fit of the spatial solutions, as measured by Stress, is summarized for the various solutions in Fig. 2. With the exception of the unidimensional solution, little difference among the three metrics occurred, and there was no clear break in the Stress curve to specify dimensionality. Consequently, the choice of a solution space was based upon psychophysical interpretability. The two-, three-, and four-dimensional solutions at each metric were examined by computing linear correlations among dimensions of the various solutions and between projections of forms on the dimensions and the values of forms on the 80 physical measures. In the case of all three metrics the four-dimensional solutions were redundant, i.e., the fourth dimension was correlated with one or more other dimensions and with form measures with which other dimensions were

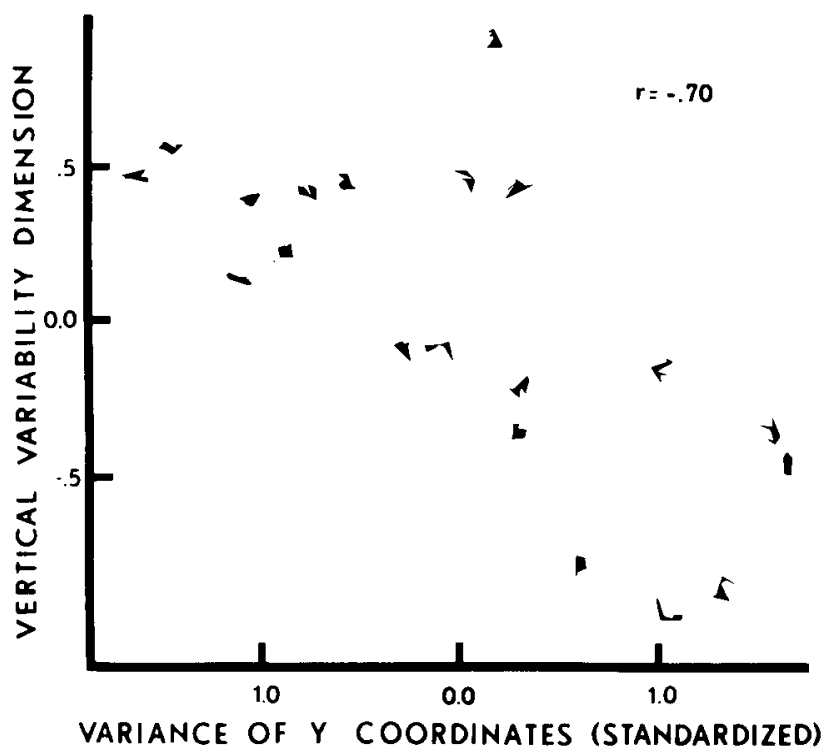

Fig. 3b. Relationship between the vertical variability dimension (similarity judgment space) and the variance of the $y$ coordinates.

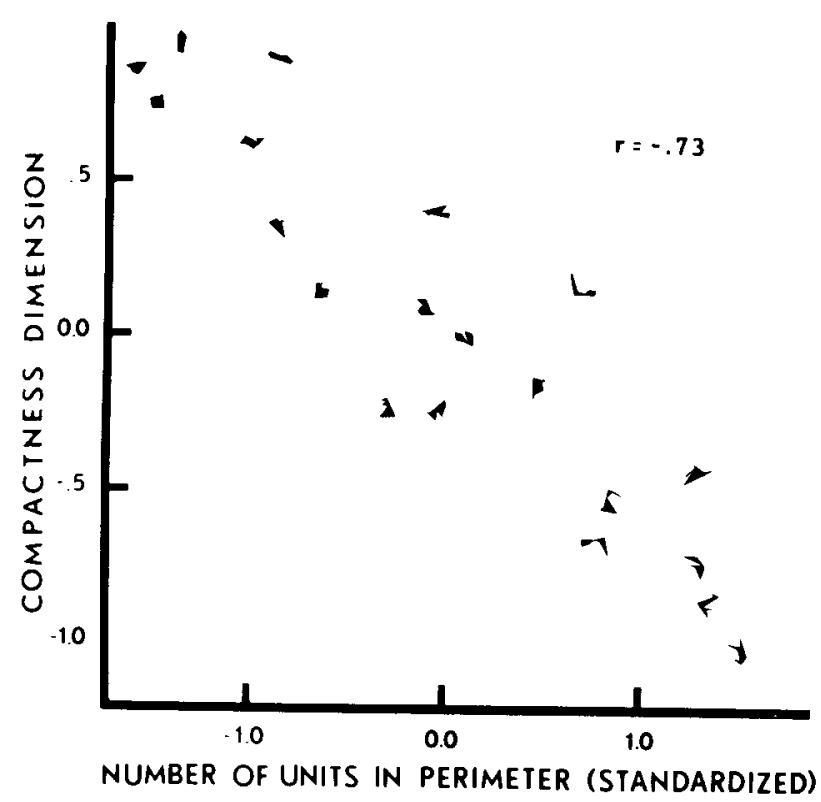

Fig. 3a. Relationship between the compactness dimension (similarity judgment space) and the number of units in the perimeter.

correlated. The solution which was maximally interpretable, the three-dimensional Euclidian solution, evidenced fair fit (Stress near $10 \%$ in Fig. 1). Figures $3 a-3 c$ show the relation between each of the dimensions and the physical measures with which they were most highly correlated, and Table 1 gives the correlations among dimensions, among measures, and between dimensions and measures. It is clear from Table 1 that the three Euclidian dimensions represent three unique orderings of the forms, since correlations among dimensions were small, and that the psychophysical fit to the measures was good.

The first dimension (Fig. 3a) was interpreted as a compactness-dispersion dimension since it was correlated with perimeter and for constant area the perimeter length is a measure of how dispersed the form is from its center of gravity. The second dimension (Fig. 3b) was labelled "vertical

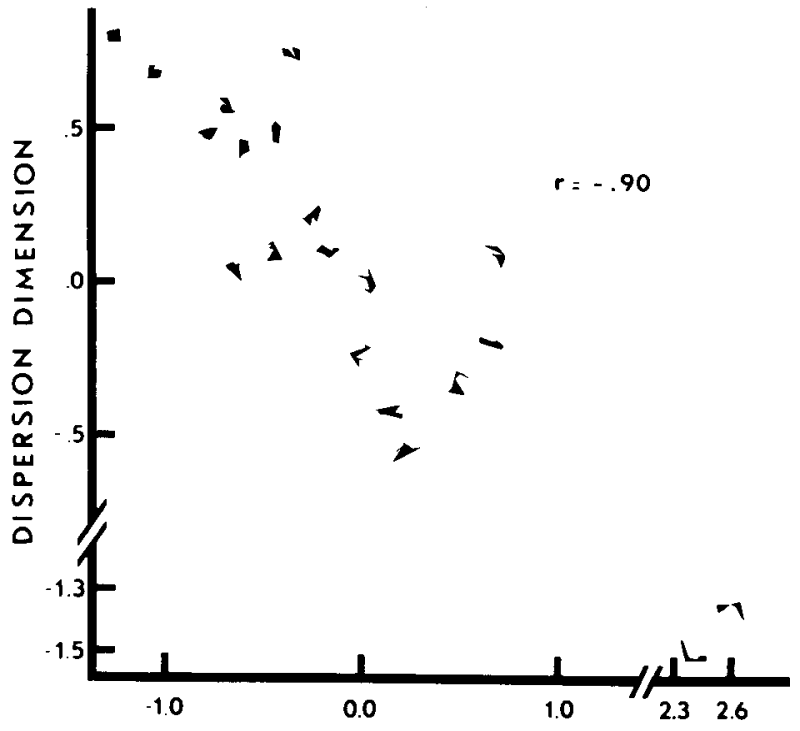

MAXIMUM SECOND AREAL MOMENT OF $X$ (STANDARDIZED)

Fig. 3c. Relationship between the dispersion dimension (similarity judgment space) and the maximum second areal moment of $x$. 
Table 1

Correlation among Dimensions of the Similarity Judgment Solution Space and between Dimensions and Measures (Decimals Omitted).

\begin{tabular}{lllllll} 
& $\mathrm{D}_{1}$ & $\mathrm{D}_{2}$ & $\mathrm{D}_{3}$ & $\mathrm{p}$ & $\mathrm{V}_{\mathrm{y}}$ & $\operatorname{Max}_{\mathrm{M} 2 \mathrm{x}}$ \\
$\mathrm{D}_{1}$ & -- & 02 & 31 & -73 & -34 & -28 \\
$\mathrm{D}_{2}$ & & -- & 25 & -33 & -70 & -33 \\
$\mathrm{D}_{3}$ & & & -- & -47 & -16 & -90 \\
\hline
\end{tabular}

$P \quad=$ Number of units in the perimeter.

$V_{y} \quad=$ Variance of $y$ coordinates.

$\operatorname{Max}_{M 2 x}=$ Maximum second areal moment of $X$.

variability" and was most highly correlated with the variance of the $y$ coordinates and was also correlated with other measures of variability with regard to the y axis (e.g., the correlation with the vertical extent, or height, of the form, was -.64). A second type of compactness-dispersion is represented in the third dimension shown in Fig. $3 \mathrm{c}$. The measure with which it was most highly correlated, the maximum second areal moment of $x$, essentially measures the extent to which the area is dispersed away from the center of gravity in any orientation. ${ }^{4}$ This dimension may be differentiated from Dimension 1 since a form may have a long perimeter due to a high degree of jaggedness while the area is not dispersed away from the center of gravity.

\section{Method}

\section{EXPERIMENT 2}

The second study was completed using the same Ss, forms, and apparatus which were used in the first experiment. Following the four days of testing in Experiment 1, Ss were required to make judgments of discrimination difficulty for the 190 pairs of forms on a seven-point scale. In particular, they were asked to judge how difficult it would be to discriminate each pair of forms if the viewing conditions were nonoptimal, i.e.; blurred, exposed for a brief duration, or viewed under low contrast conditions. Judgments were made in one session, in blocks of 50 trials, with balancing accomplished with a 4 by 4 Latin square. Reliability was not checked since all judgments were made in a single session.

\section{Results}

Mean ratings served as input to the nonmetric MDS program (Kruskal, 1964a, b) and, as in the first study, spatial configurations were computed through six dimensions with $r=1.5,2.0$, and 3.0. Goodness of fit (Fig. 4) was similar to that obtained in the first study, there was no difference among

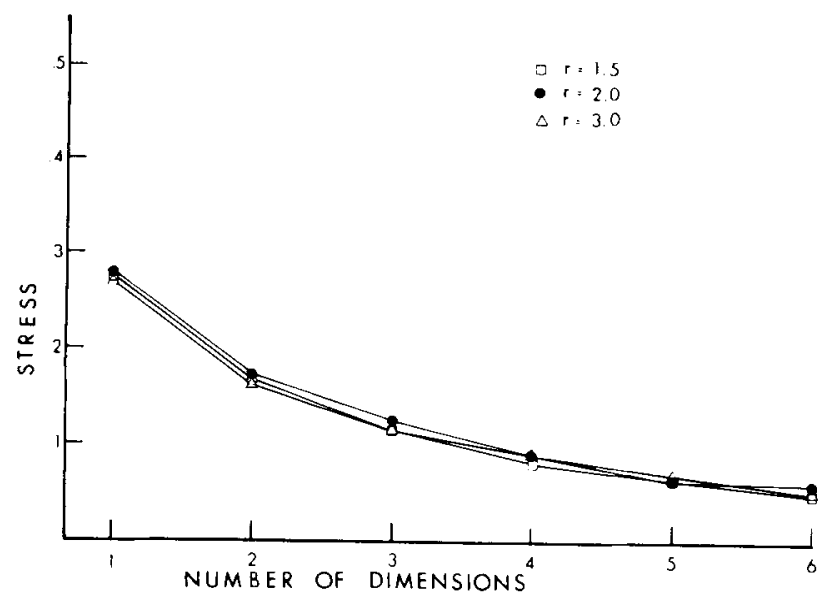

Fig. 4. Values of stress for the three Minkowski solutions $(r=1.5,2.0$, and 3.0 ) to the discrimination difficulty judgments.
Table 2

Correlation among Dimensions of the Discrimination Difficulty Judgment Solution Space and between Dimensions and Measures (Decimals Omitted).

\begin{tabular}{lccccrr}
\hline & $\mathrm{D}_{1}$ & $\mathrm{D}_{2}$ & $\mathrm{D}_{3}$ & $\operatorname{Max}_{\mathrm{MA} 3 \mathrm{x}}$ & $\mathrm{M}_{2 \mathrm{y}}$ & $\mathrm{M}_{3 \mathrm{x}}$ \\
$\mathrm{D}_{1}$ & -- & -21 & -30 & 95 & 38 & 04 \\
$\mathrm{D}_{2}$ & & - & 28 & -36 & -73 & -52 \\
$\mathrm{D}_{3}$ & & & -- & -32 & -22 & -70 \\
\hline
\end{tabular}

$\operatorname{Max}_{M A 3 X}=$ Maximum absolute third areal moment of $X$.

$M_{2 y}=$ Second areal moment of y at 0 deg rotation

$M_{3 x} \quad=$ Third areal moment of $X$ at 0 deg rotation

metrics, no sharp break in Stress occurred, a fair fit was indicated for three or four dimensions, and the level of Stress for all solutions was similar to that obtained for the similarity judgments. Accordingly, psychophysical interpretability again served as a basis for selecting a final configuration.

Correlations computed among the dimensions showed that the fourth dimension shared common variance with dimensions obtained in solutions of lower order for all metrics. Moreover, the three-dimensional Euclidian solution yielded a third dimension which was not independent of the first two dimensions. Since the $r=3$ solution in three dimensions was interpretable it was selected as the final configuration. Table 2 gives the correlations among dimensions and measures with which they are most highly correlated. The corresponding psychophysical plots are shown in Fig. 5a-5c. The first dimension (Fig. 5a) was interpreted as a dispersion dimension of the same type as the third dimension of the similarity space. The maximum absolute third areal moment, with which it was correlated .95 , is computed in the same way as the maximum second areal moment, except that third moments take both positive and negative values as the form is rotated and the sign is ignored. The second dimension (Fig. 5b) was interpreted as a "vertical skew" dimension and indicates the degree to which the area of the form is skewed below or above the center of gravity with regard to the $y$ axis of the form. Similarly, the third dimension (Fig. 5c) represents "horizontal skew" and measures distribution of area with regard to the $\mathrm{x}$ axis.

\section{CONGRUENCE AMONG SOLUTIONS}

Congruence among spatial solutions was examined through canonical correlation analyses. These techniques have been discussed in a number of multivariate statistics sources (e.g., Cooley \& Lohnes, 1962; Anderson, 1958; Morrison, 1967), but have rarely been used in perceptual research (see Stenson, 1968 , for an exception). For present purposes we may view canonical correlation as a generalization of the multiple regression problem in which a linear combination of a set of variates (predictors) is formed in such a way that the correlation between that linear composite and some single criterion variable is maximized. In the case of two sets of variates, we may form linear composites of the first set and of the second set in such a way that the correlation between these composites (canonical variates) is maximized. The number of orthogonal sets of canonical variates will be equal to the rank of the smaller rank of the two original variate matrices. The squares of the canonical correlations may be interpreted as the per cent of variance common to their respective canonical variates. In addition, the weights (canonical coefficients) which define the linear composites indicate the relative contribution of the original variates to each canonical variate.

Canonical analyses comparing the similarity judgment space, the discrimination-difficulty judgment space, and a discrimination-in-noise space presented elsewhere (Aiken \& Brown, 1968) are summarized in Tables 3, 4, and 5. The summary in Table 3 represents a comparison of the two judgment spaces. Since each of the sets of variates consisted of 


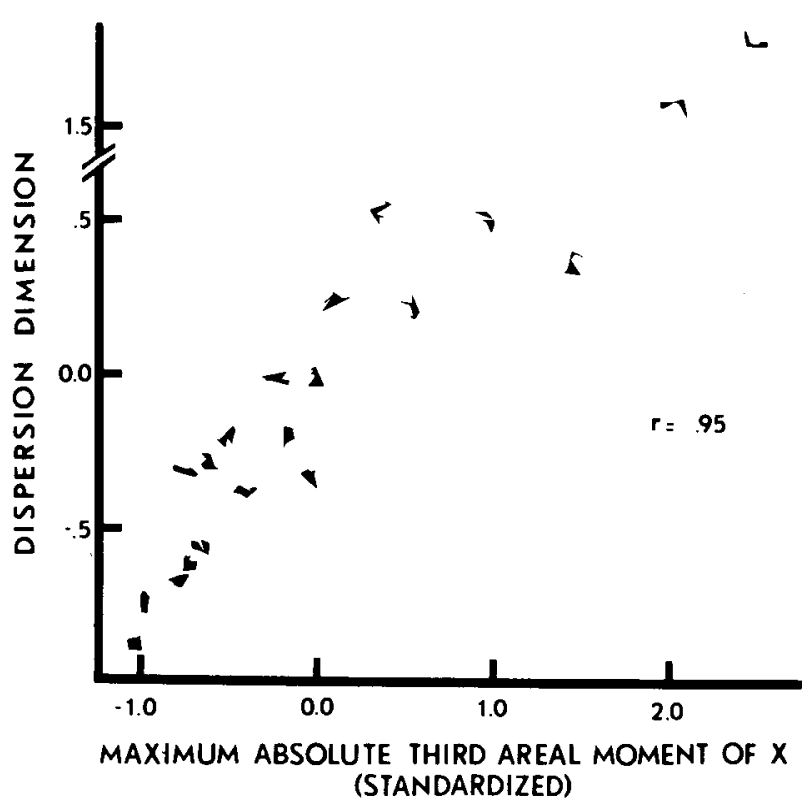

Fig. 5a. Relationship between the dispersion dimension (d iscrimination difficulty judgment space) and the maximum absolute third areal moment of $x$.

three dimensions there could be a maximum of three important canonical relations. Ideally, each set of canonical variates would weight one dimension highly from each solution and the canonical correlation would be large for each set. The first set is obviously almost completely determined by the dispersion dimensions from the two solutions and the correlation shows that these two variates share $90 \%$ common variance. The second set of variates are largely determined by distribution of the shapes with regard to the $y$ axes, but the common variance is only $38 \%$. As expected, since there is negligible correlation between dimensions within each solution, the third sets of variates weight the remaining dimension from each solution but they share only $1 \%$ variance. A summary comparison, representing the total variance shared by the two spaces, may be obtained by summing and squaring the canonical correlations and dividing by three (the total normalized variance of the variate spaces for a solution). This computation shows that the two spaces shared $46 \%$ variance.

The second comparison, summarized in Table 4 for the similarity judgments and the spatial analysis of discrimination in noise, yielded only one important relation. A linear combination of the horizontal variability dimension with less weight assigned to the side-length dimension for the discrimination data was correlated significantly with a combination of the two dispersion dimensions from the similarity judgments. The total variance shared by the two variate spaces was $21 \%$.

The last comparison (Table 5) yielded one relatively large canonical correlation, but is difficult to interpret since all dimensions in both solutions received some weight. The total common variance between these two spaces was .26 .

The tests of significance summarized in Tables 3,4 , and 5 are based upon Bartlett's approximation (Cooley \& Lohnes, 1962) for all canonical correlations since there exists a direct test only for the first canonical variates in each solution. These tests require the assumption of multinormality and information regarding robustness is not available. These tests should, therefore, be interpreted cautiously. In summary, it seems clear that the two judgment spaces were closely related in terms of two dimensions of the respective solutions. When the two judgment spaces are compared to the experimental task,

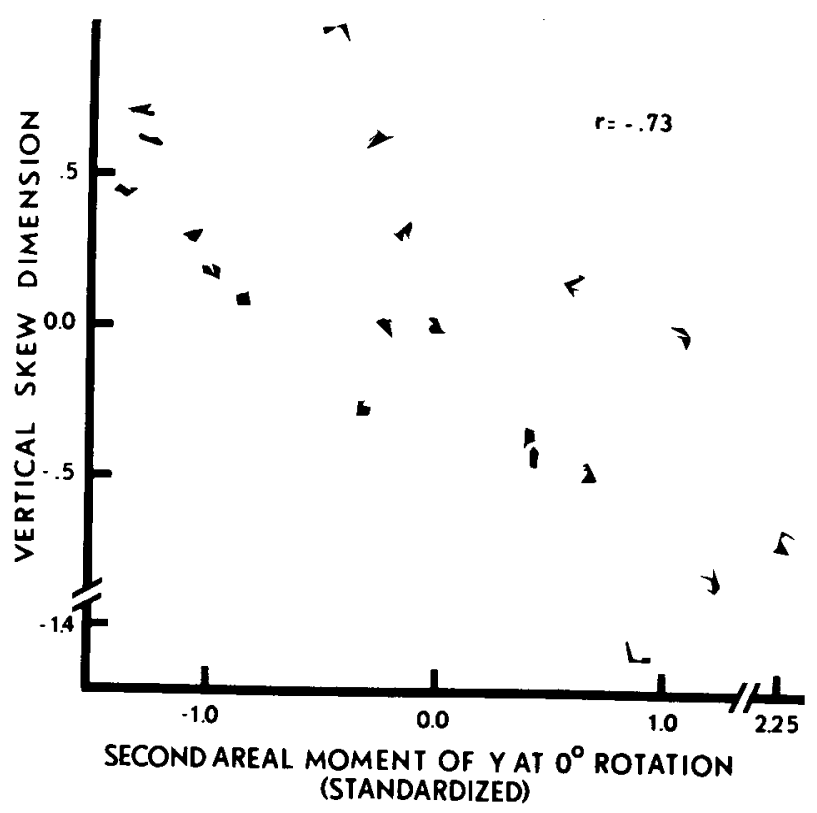

Fig. 5b. Relationship between the vertical skew dimension (discrimination difficulty judgment space) and the second areal moment of $y$ at 0 -deg rotation.

however, there is weak correspondence and interpretation of the single canonical variate for each solution is not compelling.

\section{DISCUSSION}

These studies were successful in demonstrating that psychophysically interpretable spatial solutions can be obtained with relatively complex visual patterns in a variety of tasks.

We have concluded that the two judgment tasks yielded similar configurations, but that neither of these tasks yielded results which were congruent with the discrimination data. This latter conclusion rests upon demonstrating that the

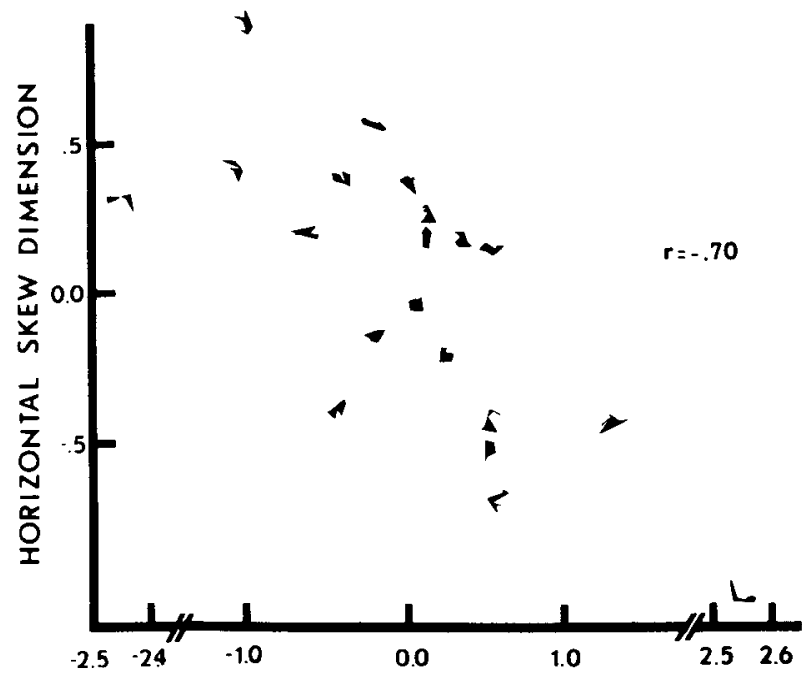

THIRD AREAL MOMENT OF $X$ AT $0^{\circ}$ ROTATION (STANDARDIZED)

Fig. 5c. Relationship between the horizontal skew dimension (discrimination difficulty judgment space) and the third areal moment of $x$ at 0 -deg rotation. 
Table 3

Canonical Coefficients ${ }^{5}$ and Correlations for a Comparison of the

Discrimination Difficulty Judgments and Similarity Judgments

\begin{tabular}{lccccccc}
\hline Canonical & & \multicolumn{2}{c}{ Discrimination Difficulty Judgments } & \multicolumn{3}{c}{ Similarity Judgments } \\
\cline { 2 - 7 } Correlation & & Dispersion & Vertical Skew & Horizontal Skew & Compactness & Vertical Variability & Dispersion \\
& & 1 & 2 & 3 & 1 & 2 & 3 \\
\hline .95 & $\mathrm{p}<.005$ & .99 & .11 & .07 & .27 & .14 & .95 \\
.62 & $\mathrm{p}<.005$ & -.21 & -.92 & -.34 & -.07 & -.92 & .39 \\
.11 & NS & .24 & -.45 & .86 & -.90 & .12 & .42 \\
\hline
\end{tabular}

Table 4

Canonical Coefficients and Correlations for a Comparison of the Discrimination Data and Similarity Judgments

\begin{tabular}{lccccccc}
\hline $\begin{array}{l}\text { Canonical } \\
\text { Correlation }\end{array}$ & \multicolumn{3}{c}{ Discrimination in Noise } & \multicolumn{3}{c}{ Similarity Judgments } \\
\cline { 3 - 7 } & & Jaggedness & Side Length & $\begin{array}{c}\text { Horizontal } \\
\text { Variability }\end{array}$ & Compactness & Vertical Variability & Dispersion \\
& & 1 & 2 & 3 & 1 & 2 & 3 \\
\hline .72 & $\mathrm{p}<.005$ & .07 & -.49 & .87 & -.39 & -.09 & .92 \\
.32 & $\mathrm{NS}$ & .94 & -.23 & .24 & .98 & .08 & .17 \\
.14 & $\mathrm{NS}$ & -.04 & .86 & .50 & .13 & .98 & .15 \\
\hline
\end{tabular}

Table 5

Canonical Coefficients and Correlations for a Comparison of the Discrimination Difficulty Judgments and Discrimination Data

\begin{tabular}{|c|c|c|c|c|c|c|c|}
\hline \multirow{3}{*}{$\begin{array}{l}\text { Canonical } \\
\text { Correlation }\end{array}$} & & \multicolumn{3}{|c|}{ Discrimination Difficulty Judgments } & \multicolumn{3}{|c|}{ Discrimination-in-Noise } \\
\hline & & Dispersion & Vertical Skew & Horizontal Skew & Jaggedness & Side Length & Horizontal \\
\hline & & 1 & 2 & 3 & 1 & 2 & 3 \\
\hline .80 & $\mathrm{p}<.005$ & -.50 & -.81 & .31 & .46 & .17 & .87 \\
\hline .38 & NS & -.81 & .59 & .06 & -.13 & -.91 & .40 \\
\hline .09 & NS & .48 & .09 & .87 & -.81 & .56 & .16 \\
\hline
\end{tabular}

discrimination results were themselves stable and meaningful. In support of this notion we may point to the psychophysical functions, the fact that the data were fairly reliable $(r=.69)$, to the selection of a solution in few dimensions, and to the high similarity between this discrimination space and another space based on discrimination latencies (Brown \& Andrews, 1968). In terms of all of these criteria, this spatial solution was not markedly different from the solutions for the other two tasks. Since they were similar, we see no reason to assume that the discrimination space differs because of error. This, then, supports the conclusion that MDS results reflect differences which are a function of specific task demands. We favor the hypothesis that observers are capable of using a wide variety of stimulus attributes to make judgments or to discriminate and that, in any given task, observers sample stimulus variation to meet task demands. It does not, then, seem reasonable to assume that one underlying variable, psychological distance, underlies all tasks. Rather, selective attention is operative where the selection process is grounded in the requirements of specific perceptual responses. Research which attempts to determine the total array of stimulus characteristics to which the observer is sensitive and the similarities and differences among them in the context of specific tasks is currently under way.

\section{REFERENCES}

AIKEN, L. S., \& BROWN, D. R. A spatial analysis of the discriminability of forms in noise. Perception \& Psychophysics, in press.

ANDERSON, T. W. Introduction to multivariate statistical analysis. New York: Wiley \& Sons, 1958.

ATTNEAVE, F., \& ARNOULT, M. D. The quantitative study of shape and pattern perception. Psychological Bulletin, 1956, 53, 452-471.
BEHRMAN, B. W., \& BROWN, D. R. Multidimensional scaling of form. A psychophysical analysis. Perception \& Psychophysics, 1968, 4 19-25.

BROWN, D. R., \& ANDREWS, M. H. Visual form discrimination: Multidimensional analyses. Perception \& Psychophysics, 1968, 3, 401-406.

BROWN, D. R., \& OWEN, D. H. The metrics of visual form: Methodological dyspepsia. Psychological Bulletin, 1967, 68, 243-259.

COOLEY, W. W., \& LOHNES, P. R. Multivariate procedures for the behavioral sciences. New York: Wiley, 1962.

FORSYTH, G. A., \& BROWN, D. R. Stimulus correlates of tachistoscopic discrimination-recognition performance: Compactness, jaggedness, and areal asymmetry. Perception \& Psychophysics, 1967, $2,597-600$.

FORSYTH, G. A., \& BROWN, D. R. Stimulus recognizability judgments as a function of the utility of physical dimensions in recognitiondiscrimination problems. Perception \& Psychophysics, 1968, 3, 35-38.

KRUSKAL, J. B. Multidimensional scaling by optimizing goodness of fit to a nonmetric hypothesis. Psychometrika, 1964a, 29, 1-27.

KRUSKAL, J. B. Multidimensional scaling: A numerical method. Psychometrika, 1964b, 29, 115-129.

MORRISON, D. F. Multivariate statistical methods. New York: McGraw-Hill, 1967.

ROSS, R. T. Optimum order for the presentation of pairs in the method of paired comparisons. Journal of Ed ucational Psychology, 1934, 25, 375-382.

SHEPARD, R. N. Similarity of stimuli and metric properties of behavioral data. In H. Gulliksen and S. Messick (Eds.), Psychological scaling: Theory and applications. New York: Wiley, 1960.

STENSON, H. H. The psychophysical dimensions of similarity among random shapes. Perception \& Psychophysics, 1968, 3, 201-214.

\section{NOTES}

1. This research was supported by Research Grant HD 00909 from the National Institute of Child Health and Human Development.

2. Address: Department of Psychology, Purdue University, Lafayette, Indiana 47907 
3. These same subjects were employed in the study presented in Aiken and Brown (1968).

4. For a more complete discussion of measures see Brown and Owen (1967). Essentially, we view the form as a distribution of area units relative to the $x$ and $y$ axes, collapse the frequency distributions of unit areas to these axes and compute momenis as for any frequency distribution. The second moment of area represents the degree to which area is dispersed when collapsed to the $\mathrm{x}$ axis. To compute the maximum value, a simulated rotation of the form in 5-deg steps is carried out, the second moment is computed in that position, and the maximum value is selected from among the 36 values. The maximum value, then, measures the maximum dispersion of area of the form from its area center of gravity.

5. The coeffjcjents have been rescaled to a common unit so that all coefficients in Tables 3,4 , and 5 may be compared.

(Accepted for publication October 7, 1968.)

\section{ERRATUM}

MAST, T. E., \& WATSON, C. S. Attention and auditory evoked responses to low-detectability signals. Perception \& Psychophysics, 1968, 4, 237-240.-Some of the detail in Figs. 3 and 4 in this article did not show in the printed article. The one on the left is Fig. 3 and the one on the right is the lefthand portion of Fig. 4.
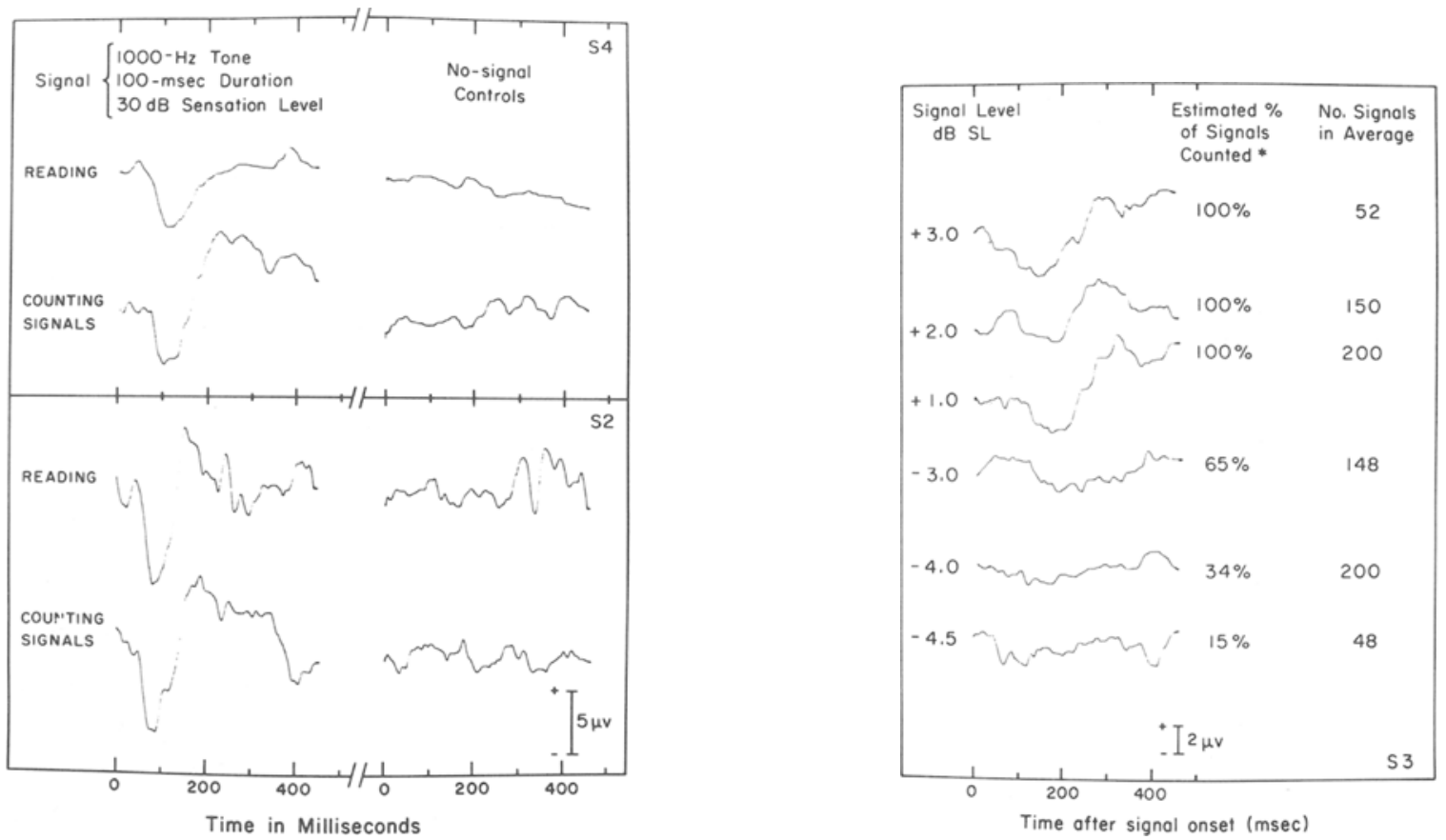